Volume 9, No.5, September - October 2020

International Journal of Advanced Trends in Computer Science and Engineering

Available Online at http://www.warse.org/IJATCSE/static/pdf/file/ijatcse127952020.pdf

https://doi.org/10.30534/ijatcse/2020/127952020

\title{
Secured Information Sharing using Data Sanitization and restoration
}

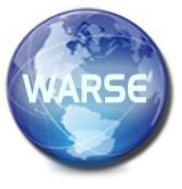

\author{
Priti Lale ${ }^{1}$, Manish Sharma ${ }^{2}$ \\ ${ }^{1,2}$ Deparment of Computer Science \& Engineering, Suresh GyanVihar University Jaipur, (RJ) India. \\ 1 priti.met@gmail.com \\ 2manish.sharma@mygyanvihar.com
}

\begin{abstract}
This paper focuses on the issues face by the supply chain management industry for overall working. Block chain technologies can contribute to the operations and supply chain domain as well. Currently, supply chains are becoming more and more complex in structure, difficult in terms of task, and diverse in terms of stakeholders, and many organizations do not have an integrated view of the entire supply chain. In supply chain management multiple stakeholders are responsible for effective communication where data sharing from vendors, suppliers, managers by maintaining transparency and privacy is very difficult. In this paper two methods are introduced for dealing with issues mention above and the methods are data sanitization and restoration.
\end{abstract}

Key words: Supply chain management, Sanitization,

Restoration, Binarization, privacy

\section{INTRODUCTION}

Information sharing has become the core activity for collaboration along the supply chain or any industry. This has been made possible by the acceptance and implantation of state-of-the-art software systems, duly supported by communication devices [3]. A number of information flows are required in a supply chain, which create a lot of value in the chain and can be viewed as the bonding agent between financial and material flows [5]. Such is the importance of information flows through the chain that a huge prohibitive cost is believed to be associated with the failure in achieving effective and efficient information flow. Blockchain technology offers many benefits, as it can provide a secure, distributed way to perform transactions among different untrusted parties.Supply chain

management is a complex process that involves taking productsmanufacturers and suppliers to consumers through a complex web of transactions. Food, beverages, clothing, and other consumer goods transform many hands before reaching consumers $[5,16]$. Implementing IoT solutions to supply chain processes allows manufacturers to monitor the status of their physical objects and capture useful data to reduce its complexities.IoT also improves transparency, traceability, security, and efficiency while reducing costs $[8,17]$.

\section{LITERATURE REVIEW}

Blockchain technology is able to transform significantly many activities and operations in the supply chain that require increasing attention from scholars and practitioners [6]. A blockchain technology enables one to enhance and track goods and passengers in real time from their origins through the overall SCM. In $[9,16]$ the supply chain, blockchain technology enables all of the actors to know who is performing which actions by defining and evidencing the time and location of the actions. Wang $\mathrm{Li}$ [1] analyzed the current situation of the domestic steel industry and steel trading enterprises, as well as the advantages and external conditions of China Railway Materials Co., Ltd., and introduced the company's efforts to strengthen resource integration, give play to its own advantages, and enhance the upstream service capacity of the steel supply chain. In $[10,14]$ block chain have four characteristics: Decentralized, Trustless, Collective Maintenance and Reliable Database. (i) Decentralized: The whole network has no central node, all nodes are equal, allowing the existence of malicious nodes, and the high robustness of block chains makes the network run stably. (ii) Trustless: In a block chain system, data can be exchanged between nodes in a trusted environment and the operation behavior is open and transparent. (iii) Collective Maintain: Data blocks in block chain system need all nodes to participate in the maintenance and (iv) Reliable Database: Every node has all the data exchange databases. Even if there is data destruction of the wrong node, the correct data can be recovered quickly [7, 17].

\section{METHODOLOGY}

Supply chain management (SCM) is an integrative concept to manage the total flows of a distribution channel. The supply chain is complex because it includes distributed activitiesfrom upstream, which deals with people, physical resources and production processes, to downstream, which covers the whole sellingprocess, i.e. contracts, sales to customers, distribution and disposal $[11,16]$. 


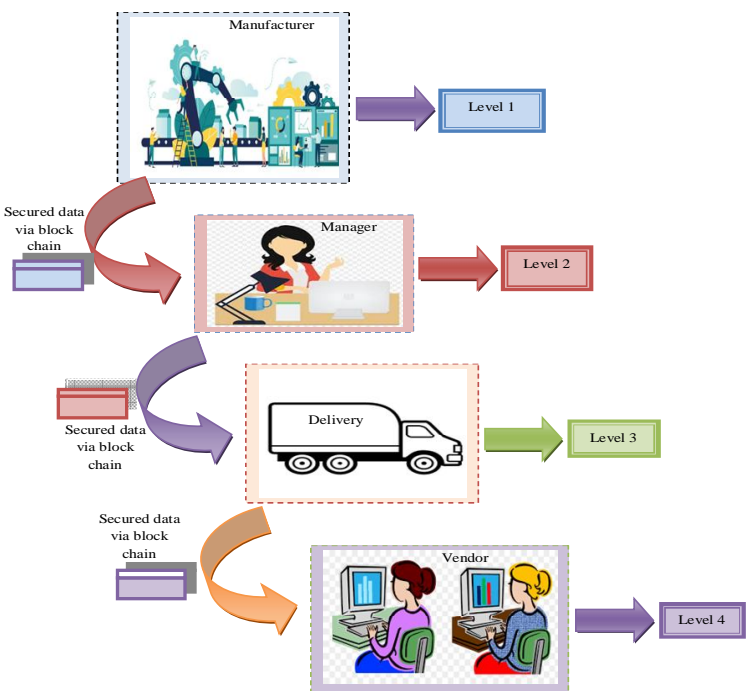

Figure 1: Supply chain management process

In supply chain management in the architecture the process is divided into four levels namely manufacturer, manager, deliver and vendor. In level 1 information about manufacturer have been stored regarding product information where sensitive fields are supposed to be send through blocks and insensitive information is kept as it is.in level 2 the same process is followed with manager through secured blockchain and it has been done for rest of the levels also. At each stage block have been added to handle security issues at every end.

\section{A. Data Sanitization}

Data sanitization is the process of hiding sensitive information in a test and development database by overwriting sensitive information with a similar type of realistic-looking but inaccurate data. Security of valuable information is basic and there are basically two types of security. The first type is concerned with the integrity of the data. In this case the revision of records is strictly controlled. For example, you cannot wish to credit or debit an account without specific controls and auditing. This type of security testing and development is not a major concern in databases. The data can be modified without any commercial impact. The second type of protection is the protection of information content from improper visibility. Names, addresses, phone numbers and credit card details are good examples of this type of data. Unlike security from updates, this type of security requires that access to information content is controlled in every environment [12, 17]. Given today's legal and commercial operating environments, most test and development databases will require some form of data hybridization. There are a variety of available technologies and usually many are needed as the format, size, and structure of the data determines.

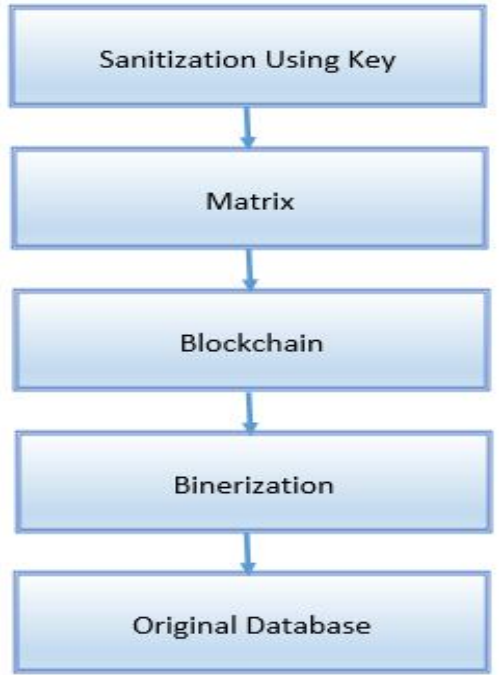

Figure 2: Data Sanitation

In data sanitization the process will temporarily destroy the sensitive data contained in initial block by implementing four processes:

\section{i. $\quad$ Sanitization using key}

In the sanitization process, the sensitive data is hidden, which prevents sensitive information from leaking while implementation. Further, the normal as well as the sensitive data is stored in blockchain fashion For the sanitization process, a key should be generated that depends on the meta-heuristic algorithm.

\section{ii. Key Matrix}

Key matrix generation process is represented in the form of matrix where matrix compromises of the block size $\mathrm{m}$ and n. and block are created at every level while transactions.

\section{iii. Binarization}

In the binarization process input is carried out in the binarised form which eventually generate sanitized blockchain $B_{1}{ }_{1}^{(n)}$ where $\mathrm{n}$ is number of hidden column. In each of block generated can be called as sanitized data where sensitive information is hidden from the intermediate level.

\section{iv. Original Database}

Original database is created with a suitable communication channel which is received by intended receiver.

\section{B. Data Restoration}

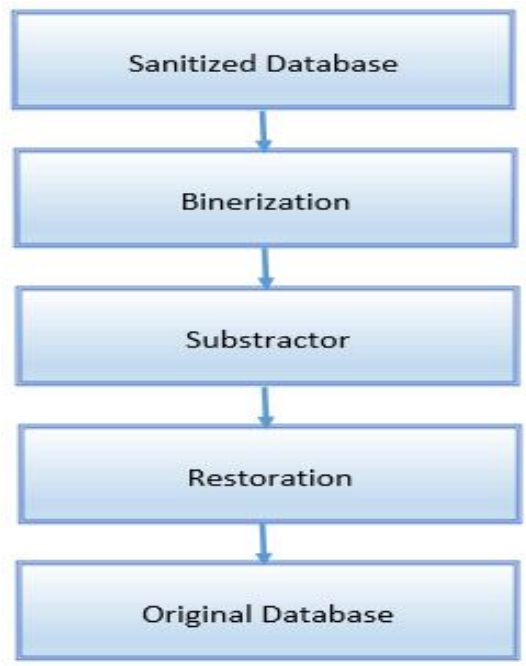

Figure 3: Data Restoration 
A data restore is the process of copying backup data from secondary storage and restoring it to its original location or a new location. A restore is done to lose data in its original state, stolen or damaged, or to transfer data to a new location.

\section{i. $\quad$ Sanitized database}

It is generated from restoration process is considered as input in restoration process. The process is executed in reverse way where again binarization is executed in which $B_{1}^{\prime \prime(n)}$ attained from sanitization and $\mathrm{K}$ generated from key matrix.

\section{ii. Binarization}

Binarization is executed attaining $\mathrm{B}_{1}^{\left({ }^{(n)}\right.}$ where represents respective blocks generated at each level and $\mathrm{n}$ is count of hidden column belongs to each level.

\section{iii. Subs tractor}

In this process the binarized key matrix and sanitized data base is use in which subtraction is implemented using adders for extracting restored database.

\section{iv. Restoration}

Restoration process is the last process where hidden data from sanitized process is restored where secured data generated at each level and it is access by only those users who are intended to use it and mediator cannot interfere between sensitive data.

\section{v. Original database}

Using data restoration original database is generated and received by only the intended user.

\section{CONCLUSION}

Data sanitization and data restoration are two proposed privacy protection procedures. Before transmission sanitized data is restored to get the original data. More importantly, through the blockchain technology, the original data were subjected to the sanitization process, where the data is converted into sanitized data. The proposed optimal key generation process is to be carried out where optimal key is identified using metaheuristic algorithm for better results.

\section{REFERENCES}

1. Ming Li, Jian Weng, Anjia Yang, Wei Lu, Yue Zhang, Lin Hou, Jia-Nan Liu, Yang Xiang, Robert H. Deng,"CrowdBC: A Blockchain-based Decentralized Framework for Crowdsourcing",IEEE Transactions on Parallel and Distributed Systems, 2018.

2. MaozhuJin, Hua Wang,Qian Zhang,Yucheng Zeng,"Supply chain optimization based on chain management mand mass customization",Information Systems and e-Business Management,October 2018.

3. AvinashSamvedi, Vipul Jain, F. T. S. Chan, S. H. Chung,"Information system selection for a supply chain based on current trends: the BRIGS approach",Neural Comput\&Applic,December 2016.

4. Scott DuHadway, Steven Carnovale,Benjamin Hazen,"Understanding risk management for intentional supply chain disruptions: risk detection, risk mitigation, and risk recovery",Ann Oper Res, march 2017.

5. Amit Karamchandani, Samir K. Srivastava, Rajiv K. Srivastavam, "Perception-based model for analyzing the impact of enterprise blockchain adoption on SCM in the Indian service
industry",International Journal of Information Management, 2019.

6. Assunta Di Vaioa, Luisa Varriale,"Blockchain technology in supply chain management for sustainable performance: Evidence from the airport industry",International Journal of Information Management, September 2019

7. FengXiong, Ruitang Xiao, Wei Ren, Rongyue Zheng\&Jianlin Jiang,"A Key Protection Scheme Based onSecretm Sharing for BlockchainBased Construction Supply Chain System",Digital Object Identifier, vol.7, August 20, 2019.

8. K. Toyoda, P. T. Mathiopoulos, I. Sasase and T. Ohtsuki, "A Novel BlockchainBased Product Ownership Management System (POMS) for Anti-Counterfeits in the Post Supply Chain", IEEE Access, vol. 5, pp. 17465-17477, 2017.

9. Aimin Yang, Yifan Li, Chenshuai Liu, Jie Li, Jiahao Wang,"Research on logistics supply chain of iron and steel enterprises based on block chain technology",Future Generation Computer Systems, Vol.101,pp635-645, December 2019.

10. Ting Chen, Derong Wang,"Combined application of blockchain technology in fractional calculus model of supply chain financial system",Chaos, Solitons \& Fractals, in communication., 2019.

11. Kay Behnke, M. F. W. H. A. Janssen,"Boundary conditions for traceability in food supply chains using blockchain technology",International Journal of Information Management, In communication, 2019.

12. Stefan Tönnissen, Frank Teuteberg,"Analysing the impact of blockchain-technology for operations and supply chain management: An explanatory model drawn from multiple case studies",International Journal of Information Management, in communication, 2019.

13. V. G. Venkatesh, Kai Kang, Bill Wang, Ray Y Zhong, Abraham Zhang,"System architecture for blockchain based transparency of supply chain social sustainability",Robotics and ComputerIntegrated Manufacturing, Vol.63,June 2020.

14. Petri Helo, A. H. M. Shamsuzzoha,"Real-time supply chain: A blockchain architecture for project deliveries",Robotics and ComputerIntegrated Manufacturing, Vol.63, June 2020.

15. Tsan-Ming Choi, Xin Wen, Xuting Sun, Sai-Ho Chung,"The mean-variance approach for global supply chain risk analysis with air logistics in the blockchain technology era",Transportation Research Part E: Logistics and Transportation Review, Vol.127,pp.178-191,July 2019.

16. M.Tawarish, Dr. K. Satyanarayana, "A Review on Pricing Prediction on Stock Market by Different Techniques in theField of Data Mining and Genetic Algorithm", Volume 8, No.1, JanuaryFebruary 2019.

17. Pawan Kumar, Prof. HariomTyagi, "Two Fold Security on Cloud CRM using Hash Encryptionand Role Based Security", Volume 9, No.4, July-August 2020. 